\title{
Impact of first wave of COVID-19 on outcomes of hospitalization for upper gastrointestinal bleeding in Hong Kong: a population- based study
}

\section{다)(1) $\odot(9$}

\author{
Authors \\ Thomas K.L. Lui, Vivien W.M. Tsui, Wai K. Leung \\ Institution \\ Department of Medicine, LKS Faculty of Medicine, \\ University of Hong Kong, Hong Kong SAR, China
}

submitted 3.7 .2020

accepted after revision 11.11 .2020

Bibliography

Endoscopy International Open 2021; 09: E284-E288

DOI 10.1055/a-1333-1337

ISSN 2364-3722

(c) 2021. The Author(s).

This is an open access article published by Thieme under the terms of the Creative Commons Attribution-NonDerivative-NonCommercial License, permitting copying and reproduction so long as the original work is given appropriate credit. Contents may not be used for commecial purposes, or adapted, remixed, transformed or built upon. (https://creativecommons.org/licenses/by-nc-nd/4.0/)

Georg Thieme Verlag KG, Rüdigerstraße 14,

70469 Stuttgart, Germany

\section{Corresponding author}

Wai K. Leung, MD, Department of Medicine, Queen Mary Hospital, University of Hong Kong, 102 Pokfulam Road, Hong Kong, China

Fax: +85228162863

waikleung@hku.hk

Supplementary material is available under https://doi.org/10.1055/a-1333-1337

\section{ABSTRACT}

Background and study aims The COVID-19 pandemic has caused a major disruption in the healthcare system. This study determined the impact of the first wave of COVID-19 on the number and outcome of patients hospitalized for upper gastrointestinal bleeding (UGIB) in Hong Kong.

Patients and methods Records of all patients hospitalized for UGIB in Hong Kong public hospitals between October 2018 and June 2020 were retrieved. The number and characteristics of patients hospitalized for UGIB after COVID-19 was compared by autoregressive integrated moving average (ARIMA) model prediction and historical cohort.

Results Since the first local case of COVID-19, there was an initial drop in UGIB hospitalizations (observed 29.8 vs predicted 35.5 per week; $P=0.05$ ) followed by a rebound (39.8 vs 26.7 per week; $P<0.01$ ) with a turning point at week 14 (Petitt's test, $P<0.001$ ). There was a negative association between the number of COVID-19 cases and the number of patients hospitalized for UGIB (Pearson correlation $-0.53, P<0.001)$. Patients admitted after the outbreak of COVID-19 had lower hemoglobin (7.5 vs baseline $8.3 \mathrm{~g} / \mathrm{dL} ; P<0.01)$ and a greater need for blood transfusion ( $64.5 \%$ vs baseline $50.4 \% ; P<0.01$ ), but similar rates of allcause mortality $(6.9 \%$ vs $7.1 \% ; P=0.82)$ and rebleeding (6.7\% vs $5.1 \% ; P=0.11)$. There was also a higher proportion of patients with variceal bleeding ( $10.5 \%$ vs baseline $5.3 \% ; P<0.01)$.

Conclusions There was a dynamic change in the number of patients hospitalized for UGIB in Hong Kong during the first wave of the COVID-19 outbreak, with more obvious impact during the initial phase only.

\section{Introduction}

The ongoing COVID-19 pandemic has posed a major challenge to the delivery of typical healthcare services worldwide. For gastroenterologists, the COVID-19 pandemic has compromised the availability of routine and even emergency endoscopy services because upper endoscopy is considered to be an aerosolgenerating procedure [1]. A study from New York, one of the epicenters of the COVID-19 pandemic, showed that cases of upper gastrointestinal bleeding (UGIB) were more severe after the COVID-19 outbreak [2]. Another Austrian study showed that the number of cases of non-variceal UGIB declined after the lockdown for COVID-19 [3].

Unlike many other countries, in Hong King, the number of COVID-19 cases remains relatively low and so far, there has been no large-scale community outbreak. However, we previously reported that the overall endoscopy volume has been reduced by more than $50 \%$ since the outbreak of COVID-19, re- 
sulting in delay in diagnosis of gastric and colorectal cancer in more than $37 \%$ of cases [4]. The aim of this study was to investigate changes in the number and characteristics of patients who were hospitalized for UGIB after the first wave of COVID19 in Hong Kong.

\section{Patients and methods}

\section{Data source and patients}

The first local case of COVID-19 was reported on January 23, 2020. From then until June 30, 2020, 1,205 COVID-19 cases were reported (the end of follow up of the present study). We identified all adult patients, aged 18 years or above, who presented with UGIB to all local public hospitals in Hong Kong between October 1, 2018 and March 31, 2020. Data were retrieved from the Clinical Data Analysis and Reporting System (CDARS), a territory-wide electronic health care database of the Hong Kong Hospital Authority. The Hospital Authority is the sole public healthcare provider for the 7.3 million local population, which manages over $85 \%$ of all hospital beds in 43 public hospitals. All local residents are eligible for heavily subsidized primary, secondary, and tertiary care provided by the Hospital Authority, which is directly funded by the government. The CDARS captures important clinical information including patient demographics, diagnosis, medication prescription and dispensing records, laboratory results, and surgery and endoscopy performed. All data in the CDARS are anonymized. The International Classification of Diseases, Ninth Revision (ICD-9), was used for disease coding and the accuracy of the coding of gastrointestinal bleeding had been verified in our previous studies [5, 6]. Patients with upper gastrointestinal bleeding (UGIB) and associated endoscopic interventions were identified with relevant ICD-9 codes (Supplementary Table 1). Other relevant clinical information including baseline demographics, endoscopic diagnosis, endoscopic intervention for hemostasis, laboratory parameters, blood transfusion requirement, 7-day rebleeding, mortality, and overall length of hospital stay were reviewed. Due to the difficulty in defining rebleeding based on the electronic database, we defined 7-day rebleeding as undergoing two upper endoscopies with the same diagnostic coding for UGIB within 7 days. Similarly, we used all-cause mortality, rather than bleeding-related mortality, in this study.

The daily number of COVID-19 new case was obtained from the Center for Health Protection of the Department of Health of Hong Kong [7]. The study was approved by the Institutional Review Board of the University of Hong Kong and the Hong Kong West Cluster of the Hospital Authority (UW 20-279).

\section{Time trend analysis of newly diagnosed upper gastrointestinal tract bleeding during COVID-19}

The week that COVID-19 started was defined as the week when the first local case of COVID-19 was diagnosed in Hong Kong, which was January 23, 2020. The end date of this study was June 30, 2020. Time trend analysis was performed on the hospitalization rates for UGIB during this period as compared with preceding months up to October 1, 2018. Because of daily and seasonal variations in UGIB, the autoregressive integrated mov- ing average model (ARIMA) was constructed to predict the number of patients with UGIB, causes of UGIB, as well as other clinical outcomes on a weekly basis. The corrected Akaike's information criterion (AICC) was used to choose the ARIMA models with different combinations of parameters. The model parameter used were a seasonal $\operatorname{ARIMA}(p, d, q)(P, D, Q)_{s}$ whereas $p, d, q$ referred to the autoregressive, difference, and moving average term of the ARIMA model and P,D,Q was the corresponding part in the seasonal part and $s$ the seasonal period. The data were first used to fit the model with adjustment for the holiday effect of Christmas and Lunar new year. The mean percentage error (MPE) was used to test the accuracy of the forecast. Sensitivity analysis was performed with Petitt's test to look for a turning point in the trend of UGIB during the whole COVID-19 study period.

\section{Statistical analysis}

The difference between the ARIMA prediction and actual number of patients diagnosed with UGIB was compared with a twosided Student's t-test. The upper and lower limit of the $95 \%$ confidence interval was used to define the worst- and bestcase scenarios in the ARIMA prediction, respectively. Other clinical parameters were compared with a Student's $t$-test when appropriate. Pearson correlation was used to assess the relationship between new COVID cases and UGIB cases with hospitalization. $P<0.05$ was considered statistically significant.

\section{Results}

\section{Observed changes in the number of UGIB cases during COVID-19}

Between October 2018 and June 2020, there were a total of 2,416 hospitalizations for UGIB, including 824 hospitalizations during the COVID-19 period. Since the first local case of COVID-19, there was a dynamic change in the number of UGIB hospitalizations, with an initial decline follow by a rebound. There was a significant negative association between the number of COVID-19 cases per week and the number of hospitalizations for UGIB per week (Pearson correlation: $-0.53, P<0.001)$. Time series analysis showed there was a turning point at week 14 from the first COVID-19 case on the trend for UGIB cases (Petitt's test, $P<0.001)$, which also coincided with the end of the first COVID-19 wave in Hong Kong ( $\mathbf{F i g . 1}$ ).

\section{Number of UGIB hospitalizations: Observed vs ARIMA model prediction}

Given potential daily and seasonal variations in UGIB incidence, we compared the observed number of UGIB hospitalizations per week with the number predicted by the $\operatorname{ARIMA}(0,1,1)$ $(0,1,1)_{52}$. The mean percentage error for the ARIMA model was $1.5 \%$.

During the initial 14-week COVID-19 period, the number of UGIB hospitalizations was significantly less than predicted (mean 29.8 vs 35.5 per week, $P=0.05$ ). However, there was a rebound in the number of UGIB hospitalizations after week 14 (observed vs predicted per week: mean 39.8 vs 26.7, $P<0.01$ ). ( $\vee$ Fig. 1 and $\triangleright$ Table 1 ) 


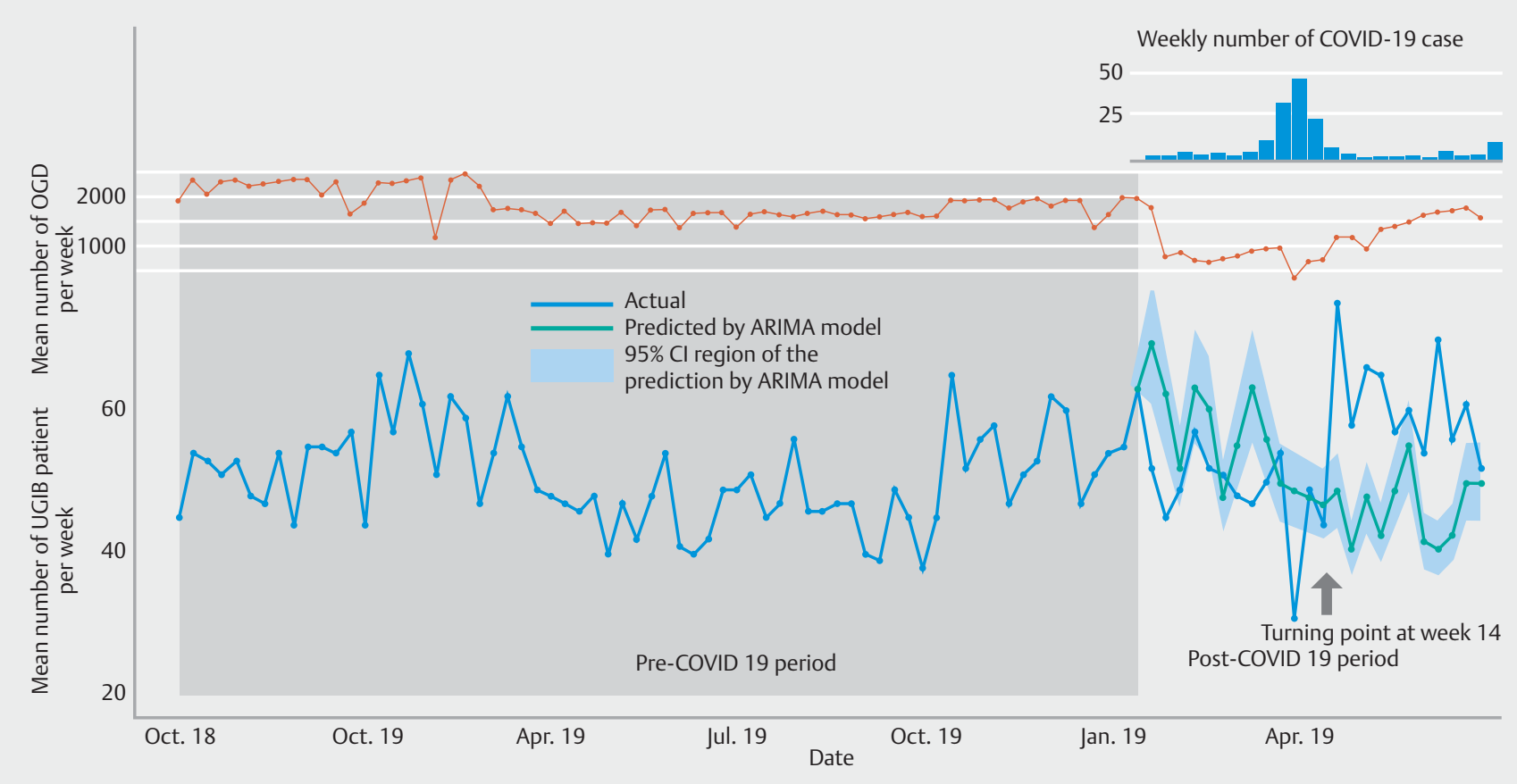

Fig. 1 Time series analysis of the number of UGIB cases after COVID-19. The upper panel showed the number of upper endoscopies (OGDs) performed between October 2018 and June 2020. The shaded area refers to the pre-COVID-19 period. The lower panel shows the number of UGIB patients hospitalized. The blue line refers to the actual number of UGIB patients observed whereas the green line is the predicted number of UGIB hospitalizations, based on the ARIMA model with $95 \% \mathrm{CI}$ (in light blue). The blue column in the top right panel shows the number of COVID-19 cases per week during the same period.

- Table1 Actual and predicted number of patients with UGIB hospitalized per week after the first local COVID-19 case.

\begin{tabular}{|l|l|l|l|}
\hline & Actual & Predicted & P value \\
\hline Overall (from Jan to Jun 2020) & $34.6[30.8-38.4]$ & $31.3[28.1-34.5]$ & 0.17 \\
\hline Before week 14 & $29.8[25.4-34.2]$ & $35.5[34.5-38.1]$ & 0.05 \\
\hline After week 14 till June 2020 & $39.8[34.5-45.1]$ & $26.7[23.6-29.8]$ & $<0.01$ \\
\hline $\begin{array}{l}\text { UGIB, upper gastrointestinal bleeding. } \\
\text { [ ], 95\% confidence interval. }\end{array}$
\end{tabular}

\section{Clinical characteristic and causes of UGIB cases during COVID-19}

The characteristics of UGIB patients admitted at different periods is shown in $>$ Table 2 . When compared to the pre-COVID-19 period, patients admitted during the COVID-19 period were significantly older (mean age 65.8 vs 67.8 years, $P=0.01$ ). For patients admitted during the COVID-19 period, the hemoglobin level was significantly lower (mean 8.3 vs $7.5 \mathrm{~g} / \mathrm{dL}, P<0.01$ ) and the blood transfusion rate was higher $(50.2 \%$ vs $64.5 \%, P<$ $0.01)$. There was no significant difference in the timing of endoscopy after admission (mean 1.02, 95\%Cl:0.98-1.05 vs $1.06,95 \% \mathrm{Cl}: 0.98-1.14$ days, $P=0.332$ ) or the percentage of patients requiring endoscopic hemostasis $(77.3 \%, 95 \% \mathrm{Cl}$ : $74.8-79.7$ vs $76.3 \%, 95 \% \mathrm{Cl}: 72.4-80.2, P=0.68)$ before and after COVID-19.
Regarding patient outcomes ( $\triangleright$ Table 3 ), there was no significant difference in rates of 7-day rebleeding (5.1\%, 95\% Cl: $3.8-$ 6.3 vs $6.7 \%, 95 \% \mathrm{Cl}: 5.2-8.3 ; P=0.11)$ or all-cause mortality (7.1\%, $95 \% \mathrm{Cl}: 5.7-8.6$ vs $6.9 \%, 95 \% \mathrm{Cl}: 4.8-8.9 ; P=0.82)$ after COVID-19. However, the average length of stay was significantly shorter after COVID-19 (11.4 vs 9.8 days, $P<0.001)$. Peptic ulcer bleeding remained the most common cause of UGIB both before (66.0\%) and after (66.1\%) COVID-19. Notably, there was a significant increase in the proportion patients with UGIB with variceal bleeding after COVID-19 (5.3\% vs $10.5 \%, P<$ $0.01)$.

\section{Discussion}

Based on the territory-wide electronic health database for Hong Kong and ARIMA prediction, we noted a dynamic change in hospitalizations for UGIB in Hong Kong. There was a small but 
- Table 2 Characteristics of patients with UGIB admitted before and after the outbreak of COVID-19 in Hong Kong.

\begin{tabular}{|c|c|c|c|}
\hline & Before COVID-19 & After COVID-19 & $P$ value \\
\hline Mean patient age in years $( \pm S D)$ & $65.8 \pm 0.5$ & $67.8 \pm 1.8$ & 0.01 \\
\hline Male sex & $68.4 \%$ & $67.8 \%$ & 0.77 \\
\hline Hemoglobin in $\mathrm{g} / \mathrm{dL}( \pm \mathrm{SD})$ & $8.3 \pm 0.1$ & $7.5 \pm 0.2$ & $<0.01$ \\
\hline Urea in $\mathrm{mmol} / \mathrm{L})( \pm \mathrm{SD})$ & $13.7 \pm 1.0$ & $12.5 \pm 0.6$ & 0.90 \\
\hline Creatinine in umol/L ( $\pm S D$ ) & $137 \pm 13$ & $163 \pm 25$ & 0.33 \\
\hline Blood transfusion & $50.2 \%[47.6-52.9 \%]$ & $64.5 \%[59.3-69.7 \%]$ & $<0.01$ \\
\hline Endoscopic hemostasis & $77.3 \%[74.8-79.7 \%]$ & $76.3 \%[72.4-80.2 \%]$ & 0.68 \\
\hline
\end{tabular}

- Table 3 Outcomes and causes of UGIB in patients admitted during the COVID-19 pandemic.

\begin{tabular}{|c|c|c|c|}
\hline & Before COVID-19 & After COVID-19 & $P$ value \\
\hline Rebleeding within 7 days & $5.1 \%[3.8-6.3 \%]$ & $6.7 \%[5.2-8.3 \%]$ & 0.11 \\
\hline All-cause mortality & $7.1 \%[5.7-8.6 \%]$ & $6.9 \%[4.8-8.9 \%]$ & 0.82 \\
\hline Length of stay in days ( \pm SD) & $11.4 \pm 0.5$ & $9.8 \pm 0.5$ & 0.02 \\
\hline \multicolumn{4}{|c|}{ Causes of UGIB (Mean number of cases per week) } \\
\hline Peptic ulcer & $20.4(66.0 \%)$ & $23.0(66.1 \%)$ & 0.13 \\
\hline Variceal & $1.6(5.3 \%)$ & $3.6(10.5 \%)$ & $<0.01$ \\
\hline Hemorrhagic gastritis & $2.3(7.4 \%)$ & $1.9(5.5 \%)$ & 0.49 \\
\hline Hemorrhagic duodenitis & $0.2(0.8 \%)$ & $0.1(0.3 \%)$ & 0.27 \\
\hline Esophageal hemorrhage & $0.2(0.7 \%)$ & $0.2(0.5 \%)$ & 0.45 \\
\hline Mallory Weiss tear & $4.8(15.5 \%)$ & $4.2(12.2 \%)$ & 0.25 \\
\hline Angiodysplasia & $0.5(1.7 \%)$ & $0.8(2.3 \%)$ & 0.22 \\
\hline Dieulafoy lesion & $0.6(1.9 \%)$ & $0.8(2.3 \%)$ & 0.29 \\
\hline Others & $0.2(0.5 \%)$ & $0.03(0.1 \%)$ & 0.40 \\
\hline
\end{tabular}

significant reduction in hospitalizations for UGIB in Hong Kong during the initial phase of COVID-19, followed by a rebound in the number of UGIB hospitalizations. Patients admitted during the COVID-19 outbreak were found to be older with lower hemoglobin levels. There was also a higher proportion of variceal bleeding during this period.

In contrast to New York and Austria [2,3], Hong Kong was never locked down during this pandemic. However, there was still a significant reduction in the volume of endoscopy performed during COVID-19 (>50\%), particularly elective procedures [4]. This study further showed that even the number of hospitalizations for UGIB was affected during the initial phase of COVID-19 outbreak. We showed that there was an inverse association between the number of COVID-19 cases and the number of UGIB hospitalizations. During the first 14 weeks of the
COVID-19 outbreak in Hong Kong, there was a significant decrease in patients presenting to the hospital with UGIB compared to the prediction from our ARIMA modelling. Notably, we observed a rebound in the number of patients with UGIB during the later phase of COVID-19. The reason for this rebound is unknown but may be due to the delay in presentation of some patients with minor bleeding. In fact, the proportion of patients with variceal bleeding actually increased during the COVID-19 period, which supports this speculation.

Although outcomes in patients who were not admitted to the hospital cannot be determined, there was no significant difference in rates of observed 7-day rebleeding and all-cause mortality, before and after COVID-19. Notably, there was a significantly shorter length of stay for patients with UGIB after COVID-19. In addition to creating space in the hospital for pa- 
tients with COVID-19, the significantly shorter stays may also have been due to patient or physician preference, possibly based on previous adverse experiences associated with the SARS outbreak in Hong Kong in 2003, when hospital-acquired infection played a major role.

The strength of this study was the use of the comprehensive local public health care database, which captured information on all patients admitted to public hospitals in Hong Kong. Moreover, as the incidence of UGIB can vary with the season and even the day of the week, we used the ARIMA model based on weekly data from 2018 to derive the predicted numbers of patients hospitalized for UGIB.

This study has limitations. First, there was a lack of proper control, particularly absence of information on patients with UGIB not hospitalized during this period or admitted to private hospitals. Second, we only looked at short-term outcome and not longer-term data. Third, limited information was available on baseline characteristics due to the use of an electronic healthcare database and the risk of misclassification based on ICD-9 codes alone. However, the same electronic database has been validated in our previous GIB studies $[6,7]$. Finally, the accuracy of adjudication of outcomes such as rebleeding and bleeding-related death cannot be verified in the electronic database.

\section{Conclusion}

In conclusion, there was a dynamic change in the number of patients hospitalized for UGIB during the first wave of COVID-19 in Hong Kong with an initial drop followed by a rebound in the number of cases. Patients admitted during COVID-19 were older with lower hemoglobin levels. There was also a higher proportion of bleeding varices during this period, but there were no observable changes in major clinical outcomes such as rebleeding and death. Our study findings suggest that the health-seeking behavior of patients who presented with UGIB may have been affected by the COVID-19 pandemic, which was inversely associated with the number of local COVID-19 cases and the trend was more prominent in the initial phase of the outbreak.

\section{Acknowledgments}

The authors thank the Hospital Authority of Hong Kong for access to data available from the Clinical Data Analysis and Reporting System

\section{Competing interests}

The authors declare that they have no conflict of interest.

\section{References}

[1] Sagami R, Nishikiori H, Sato T et al. Aerosols produced by upper gastrointestinal endoscopy: a quantitative evaluation. Am J Gastroenterol 2020: doi:10.14309/ajg.0000000000000983

[2] Kim J, Doyle JB, Blackett JW et al. Effect of the Coronavirus 2019 Pandemic on outcomes for patients admitted with gastrointestinal bleeding in New York City. Gastroenterology 2020; 159: 1155-1157, e1151

[3] Schmiderer A, Schwaighofer $H$, Niederreiter $L$ et al. Decline in acute upper gastrointestinal bleeding during Covid-19 pandemic after lockdown in Austria. Endoscopy 2020; 52: 1036-1038

[4] Lui TKL, Leung K, Guo CG et al. Impacts of the Coronavirus 2019 Pandemic on gastrointestinal endoscopy volume and diagnosis of gastric and colorectal cancers: a population-based study. Gastroenterology 2020; 159: 1164-1166, e1163

[5] Guo CG, Cheung KS, Zhang F et al. Incidences, temporal trends and risks of hospitalisation for gastrointestinal bleeding in new or chronic low-dose aspirin users after treatment for Helicobacter pylori: a territory-wide cohort study. Gut 2020; 69: 445-452

[6] Chan EW, Lau WC, Leung WK et al. Prevention of dabigatran-related gastrointestinal bleeding with gastroprotective agents: a populationbased study. Gastroenterology 2015; 149: 586-595, e583

[7] The Center for Health Protection of the Department of Health of Hong Kong. COVID-19 theme website. Verfügbar unter: https://www. coronavirus.gov.hk/eng/index.html 